\title{
Image Edge-Segmentation Techniques : A Review
}

\author{
Rana Riad K. Al-Taie*, Basma Jumaa Saleh, Lamees Abdalhasan Salman
}

Department of Computer Engineering/ Al-Mustansiriyah University/ Baghdad, Iraq

\section{Article Info \\ Volume 8, Issue 5 \\ Page Number : 252-257}

\section{Publication Issue :}

September-October-2021

\section{Article History}

Accepted : 01 Oct 2021

Published: 10 Oct 2021

\begin{abstract}
Image segmentation is commonly applied technique in different domains such as automatic pattern recognition, image retrieval based content, machine vision, face detection, medical imaging, and object detection. Image segmentation involves classifying or identifying sub patterns in a given image. Many of algorithms and techniques for image segmentation have been proposed to optimize segmentation problems in a specific application area. In this work, different image segmentation techniques had been applied (threshold based, region based segmentation and edge based preserving methods. This Experiment have been done using MATLAB R2018b. Different edge detection methods such as Sobel, Prewitt, Roberts, Laplacian, Kiresh and Canny methods are performed on the benchmark image and the performance is analyzed with respect to the standard measure peak signal-to-noise ratio (PSNR), and mean square error. The results present that the Laplacian method is more effective than the other methods.
\end{abstract}

Keywords : Segmentation, Edge-Preserving, Image Processing

\section{INTRODUCTION}

Computer vision has become an essential component of engineering Image segmentation is an essential part of many image analysis applications. Most of the procedures need to be fragment into image processing. Based on a pre-defined principle, image segmentation is produced to fragment the image into areas. Particularly, we might conclude that segmentation is a mechanism to demarcate the foreground area from the context. In terms of specific characteristics such as colour, texture, intensity, the different areas are homogeneous [1]. It remains a difficult problem to extract the region of interest from the picture. The resulting image processes such as edge-preserving, image segmentation, and object detection may be poor if the input image involves noise [2]. The noise given in the image together with the uncertainty of the image files is among the major segmentation challenges. Choosing an appropriate methodology for segmentation is a complicated problem. It is a difficult challenge to allocate pixels to the segment of symbols. Centrist, the number of sub-fragment to be executed. Education images used algorithms based on classification. Photo fragmentation discovers its 
implementations in image categorization actual measurements of shape and size. Conversely, image restoration structures based on content to take part in the segmentation mechanism [3]. The edgepreserving system dramatically decreases the number of data and filters out pointless drivel while preserving important compressive strength in an image. Edge preserving is a technique that detects the points where the brightness of the object varies dramatically or formally.

\section{IMAGE- SEGMENTATION}

This first stage in the analysis of the image is the portion of the image. Divide a picture into bits or items that travel up. The degree to which this subdivision is taken, depending on the issue being considered. Sometimes the item needs to be segmented from the context to interpret the approach based on segmentation, discontinuity, and similarity. One approach is to reformat an image depends on the first method are sudden gray-level image changes and can be divided into three approaches: (1) point detection, (2) line detection, and (3) edge preservation. The second algorithm is a method based on finding an image's identical region based on the region's growth and threshold. This work uses the Edge-preserving technique to discuss the first algorithm [5].

\section{EDGE-PRESERVING}

Edge-based methods of segmentation depend on shape, black, colour of boundaries. based on discontinuities. Edge-preserving is a common problem with undefined boundaries where gaps exist on the edges [3].Fake edge-preserving problems, Lack of real edges, slim or stiff lines and problems with noise. In this article, we tested and made a graphical summary of the most commonly utilized edgepreserving techniques centered on gradient and Laplacian for the challenges of inaccurate edge- preservation [6]. Using MATLAB 2019b, the software is developed. It is a series of actions aimed at identifying points in a picture where there are clearly defined adjustments in frequency. This sequence of acts is necessary to understand the value of the content of an object and its applications in image and machine vision evaluation. The final use of a clear and de described a variation in image brightness discovery is to reflect the final activities and adjustments in the world's material properties [7]. Edge preservation is a basic issue in the analysis of images. Edges define the borders of objects and are therefore useful for segmenting, marking and distinguishing objects [8]. Specific edge-preservations are:

\section{A. Sobel-edge-preserving}

Sometimes referred to as the Sobel-Feldman technique or Sobel operator, the technique of Sobel is applied at image analysis and computer detection, especially in edge-preserving techniques where edges are underlined [9]. The Sobel-Feldman algorithm is based on converting the image with a thin, divisible, and integral quality of filter in vertically and horizontally dimensions. The estimation of the differential it generates is crude, especially for highfrequency image diversities [10]. In digital images, the computation of the partial gradient derivation can be approximated Use the technique of Sobel displayed in the masks elsewhere here:

\begin{tabular}{|c|c|c|}
\hline 1 & 2 & 1 \\
\hline 0 & 0 & 0 \\
\hline-1 & -2 & -1 \\
\hline
\end{tabular}

\begin{tabular}{|c|c|c|}
\hline-1 & 0 & 1 \\
\hline-2 & 0 & 2 \\
\hline-1 & 0 & 1 \\
\hline
\end{tabular}

Figure 1. Sobel-edge-preserving mask.

At each point of the image, the computational correspondence can be connected to produce the differential and amplitude of the standard image [5], using: 


$$
\begin{array}{r}
|\nabla f|=\sqrt{S_{i}^{2}+S_{j}^{2}} \\
|\nabla f|=\left|S_{i}\right|+\left|S_{j}\right|
\end{array}
$$

\section{B. Prewitt-edge-preserving}

Except that the constant $\mathrm{c}=1$. The technique of Prewitt utilizes the like formulas as the technique of Sobel. Hence: Note that, unlike the technique of Sobel, this technique does not put any focus on pixels adjacent to the mask middle. The technique of Prewitt is evaluating two elements. The vertical-edge part is determined utilizing the Pi kernel and the horizontal-edge-portion is determined utilizing the $\mathrm{Pj}$ kernel. This shows the gradient intensity and the current pixel magnitude [5].

$$
\begin{aligned}
& |\nabla f|=\sqrt{P_{i}^{2}+P_{j}^{2}} \\
& |\nabla f|=\left|P_{i}\right|+\left|P_{j}\right|
\end{aligned}
$$

And the mask of this method is:

\begin{tabular}{|c|c|c|}
\hline 1 & 1 & 1 \\
\hline 0 & 0 & 0 \\
\hline-1 & -1 & -1 \\
\hline
\end{tabular}

\begin{tabular}{|c|c|c|}
\hline-1 & 0 & 1 \\
\hline-1 & 0 & 1 \\
\hline-1 & 0 & 1 \\
\hline
\end{tabular}

Figure 2. Prewitt-edge-preserving mask

\section{Roberts-edge-preserving}

The Robert Cross algorithm performs a clear, easy-tocalculate, two-dimensional spatial differential analysis on an object. Pixel values are the approximate absolute magnitude of the test image's spatial gradient at that position at each position in the picture. The operator comprises a couple of two ${ }^{*}$ two multiplicity kernels as shown in Figure 2. One kernel is the only one to be flipped by $90^{\circ}$ [ 5 ]. It is very close to Sobel's method.

\begin{tabular}{|l|l|}
\hline 1 & 0 \\
\hline 0 & -1 \\
\hline
\end{tabular}

\begin{tabular}{|l|l|}
\hline 0 & 1 \\
\hline-1 & 0 \\
\hline
\end{tabular}

Figure 3.Roberts-edge-preserving mas

These kernels are structured to respond to the total edges operating as $45^{\circ}$ for the grid of pixel, a kernel for every one of the pair perpendicular acclimation. The kernels can be appropriate independently to the test image, the resulting approximations to give the gradient and magnitude of the original image using:

$$
\begin{gathered}
|\nabla f|=\sqrt{R_{i}^{2}+R_{j}^{2}} \\
|\nabla f|=\left|R_{i}\right|+\left|R_{j}\right|
\end{gathered}
$$

Canny's classic technique has excellent edgepreserving efficiency, position, and a single edge reaction. The edge detector of the Canny follows the steps below, (a) first use a nonlinear filter to soften the image to remove the noise. (B) Using the Sobel operator, the image slop is then used to emphasize areas. (C) Followed by the deletion of any nonmaximum pixels. (D) The available unrestricted pixels are traced with hysteresis [10 ]. Double steps method uses two Ț1 and Ț2 thresholds to classify the slop into three groups;

- Slops > Ț2: an edge value.

- Slops < T,1: probably a co-edge value.

Therefore the result will be made based on the point of angels and the current edge directions. These are the algorithmic guidelines for the strategy of canny edge-preserving: 
1) . Image of convolution $f(r, c)$ to obtain soft image $f^{\wedge}(r, c)$ with a Gaussian function. $F^{\wedge}(r, c)=(r, c)^{*} G(r$, $\mathrm{c}$,$) .$

2) 2).To measure the edge power, first apply the difference gradient operator, then the edge scale and angle are produced as now.

3) Enable repression of non-maximum and essential magnitude.

4) Use the non-maximum suppression photo limit.

\section{A. Kiresh-edge-preserving,}

Kirsch's proposed edge-preserving method seeks peak edge intensity in two predetermined directions. The technician takes a quick kernel mask and rotates in 45 incremental steps via all 0 to 8 angles of the method (i.e. $\check{N}, \hat{S}, \breve{E}, \hat{W}, \breve{N} \hat{W}, \hat{S} \hat{W}$, ŃĚ, ŜĚ). In all dimensions, the edge-magnitude is calculated as the peak [10]. The pixels of images use these eight-masking's angels to convert, each masking has a good reply to an indisputable direction of the angel, and the output value of this point is set to the highest value of all eight angles. The masking series ' highest result number is the edge path code [12]. The generator selects one kernel mask and rotates it in increments of 45 degrees through all 8-axis directions: $\check{N}$, Ś, $\breve{E}, \hat{\mathrm{W}}$, ŇŴ, ŜŴ, ŃĚ and ŜĚ.

$$
H_{L, M}=\operatorname{Max}_{Z=1, \ldots, 8} \sum_{x=-1}^{1} \sum_{y=-1}^{1} K_{x y}^{Z} \cdot F_{L+x, M+y}
$$

Where $\mathrm{z}$ enumerates the compass direction kernels $\mathrm{K}$, kirsch operator represented by the mask [14]:

$$
\begin{aligned}
K_{N} & =\left[\begin{array}{ccc}
5 & 5 & 5 \\
-3 & 0 & 5 \\
-3 & -3 & -3
\end{array}\right], \\
K_{S} & =\left[\begin{array}{ccc}
-3 & -3 & -3 \\
-3 & 0 & -3 \\
5 & 5 & 5
\end{array}\right], \\
K_{E} & =\left[\begin{array}{ccc}
-3 & -3 & 5 \\
-3 & 0 & 5 \\
-3 & -3 & 5
\end{array}\right],
\end{aligned}
$$

$$
\begin{gathered}
K_{W}=\left[\begin{array}{ccc}
5 & -3 & -3 \\
5 & 0 & -3 \\
5 & -3 & -3
\end{array}\right], \\
K_{N W}=\left[\begin{array}{ccc}
5 & 5 & -3 \\
5 & 0 & -3 \\
-3 & -3 & -3
\end{array}\right], \\
K_{S W}=\left[\begin{array}{ccc}
-3 & -3 & -3 \\
5 & 0 & -3 \\
5 & 5 & -3
\end{array}\right], \\
K_{N E}=\left[\begin{array}{ccc}
-3 & 5 & 5 \\
-3 & 0 & 5 \\
-3 & -3 & -3
\end{array}\right], \\
K_{S E}=\left[\begin{array}{ccc}
-3 & -3 & 5 \\
-3 & 0 & 5 \\
-3 & 5 & 5
\end{array}\right],
\end{gathered}
$$

Figure 4. Kiresh-edge-preserving mask

\section{B. Laplacian of Gaussian-edge-preserving}

C. This LOG operator smoothest the image with the Gaussian-shaped kernel through convolution, followed by the Laplacian operator [13]. Gaussian edge-preserving mask Laplacian is:

$$
L_{x}=\left[\begin{array}{ccc}
0 & -1 & 0 \\
-1 & 4 & -1 \\
0 & -1 & 0
\end{array}\right]
$$

$$
\text { And } L_{y}=\left[\begin{array}{ccc}
-1 & -1 & -1 \\
-1 & 8 & -1 \\
-1 & -1 & -1
\end{array}\right]
$$

A 2nd-order differential is the image Laplacian $G$ (m, $k$ ) described as:

$$
\Delta^{2} G=\frac{\partial^{2} G}{\partial m^{2}}+\frac{\partial^{2} G}{\partial k^{2}}
$$

\section{Simulation Experiment and Performance} Estimation

Below are the simulation experiments are performed on a classical computer with an AMD A8-4500M APU with Randeon ${ }^{\text {TM }}$ HD graphics $1.9 \mathrm{GHz}$ 6.00GB RAM, 64-bit operating system, and MATLAB 2019b to comparative experiment for different edgepreserving techniques (Figure 5). The work takes the 
peak signal to noise ratio (PSNR) and the mean square error (MSE) between the preserving- image and the original image, the performance estimation index was chosen. The definition of PSNR and MSE are at, (9) and (10), respectively [15].

$$
\text { PSNR }=20 \log _{10}\left(\frac{255}{\sqrt{M S E}}\right)
$$

$$
M S E=\frac{\sum_{i=1}^{M C} \sum_{j=1}^{W}\left\|y_{i, j}-\tilde{y}_{i, j}\right\|^{2}}{3 * M * \mathcal{N}}
$$

We present the data of two algorithms experimentations in TABLE 1.

\section{TABLE 1}

COMPARISON OF THE SIX ALGORITHMS ' RESULTS

\begin{tabular}{|l|l|l|}
\hline techniques & PSNR & MSE \\
\hline Sobel-edge-preserving & 7.6561 & $1.3719 \mathrm{e}+04$ \\
\hline Prewitt-edge-preserving & 7.6561 & $1.3719 \mathrm{e}+04$ \\
\hline Roberts-edge-preserving & 7.6555 & $1.3720 \mathrm{e}+04$ \\
\hline log-edge-preserving & 7.6575 & $1.3716 \mathrm{e}+04$ \\
\hline Canny-edge-preserving & 7.6588 & $1.3713 \mathrm{e}+04$ \\
\hline kirsch-edge-preserving & - & $8.8085 \mathrm{e}+04$ \\
& 1.2421 & \\
\hline
\end{tabular}

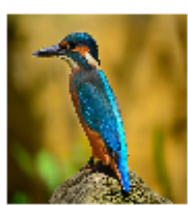

(a)

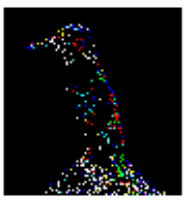

(b)

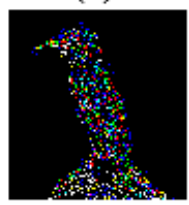

(e)

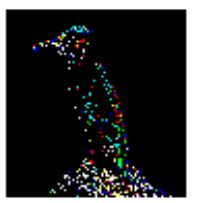

(c)

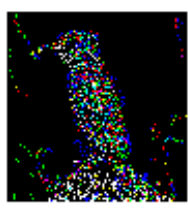

(f)

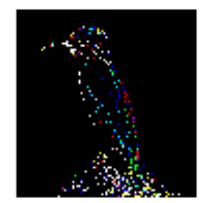

(d)

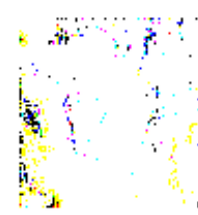

(g)
Figure 5. Comparative experiment for different edgepreserving techniques, (a) Benchmark image, (b)
Sobel-edge-preserving, (c) Prewitt-edge-preserving, (d) Roberts-edge-preserving,(e)log-edge-preserving, (f)Canny-edge-preserving, (g)kirsch-edge-preserving.

\section{CONCLUSION}

A variety of image-preserving methods of segmentation has been explored in this work. It includes the area of object recognition, computer vision, image processing, and is widely used for medical images, character recognition, etc. For the edge-preserving technique, the inspired optimization algorithms have also been used recently. A brief general edge-preserving technique division is given and tested for understanding these techniques on the benchmark (bird) image Figure 5.a The results of the experiment demonstrate that implementing of logedge-preserving and Canny-edge-preserving techniques in this work is better than the other techniques it has significant advantages, especially at high peak-signal-to-noise criteria and low squared error. Therefore, maintaining the edge as a connection between the levels of the image. As new invention develops, these calculations can be improved for continuous application in different fields such as medical, object location, X- radiology and many more.

\section{ACKNOWLEDGMENT}

The authors would like to thank Mustansiriyah University (www.uomustansiriyah.edu.iq) BaghdadIraq for its support in the present work.

\section{REFERENCES}

[1]. Venmathi, A. R., Ganesh, E. N., \& Kumaratharan, N. (2016). Kirsch compass Kernel edge detection algorithm for micro 
calcification clusters in mammograms. MiddleEast Journal of Scientific Research, 24(4), 15301535. .https://doi.org 10.5829/idosi.mejsr.2016.24.04.23384.

[2]. Saleh, B. J., Saedi, A. Y. F., al-Aqbi, A. T. Q., \& abdalhasan Salman, L. (2021). Optimum Median Filter Based on Crow Optimization Algorithm. Baghdad Science Journal, 18(3), 0614-0614. https://doi.org/10.21123/bsj.2021.18.3.0614

[3]. Da Rugna, J., Chareyron, G., \& Konik, H. (2011, October). About segmentation step in contentbased image retrieval systems. In World Congress on Engineering and Computer Science (pp. 550-554). https://doi.org/10.1007/978-3319-69137-4_17

[4]. Dhankhar, P., \& Sahu, N. (2013). A review and research of edge detection techniques for image segmentation. International Journal of Computer Science and Mobile Computing, 2(7), 86-92.

[5]. Maini, R., \& Aggarwal, H. (2009). Study and comparison of various image edge detection techniques. International journal of image processing (IJIP), 3(1), 1-11.

[6]. Biswas, R., \& Sil, J. (2012). An improved canny edge detection algorithm based on type-2 fuzzy sets. Procedia Technology, 4, 820-824. https://doi.org/10.1016/j.protcy.2012.05.134

[7]. Mageswari, S. U., Sridevi, M., \& Mala, C. (2013). An experimental study and analysis of different image segmentation techniques. Procedia engineering,

64. https://doi.org/10.1016/j.proeng.2013.09.074

[8]. Al-Amri, S. S., Kalyankar, N. V., \& Khamitkar, S. D. (2010). Image segmentation by using edge detection. International journal on computer science and engineering, 2(3), 804-807.

[9]. https://en.wikipedia.org/wiki/Sobel_operator.

[10]. Singh, S., \& Datar, A. (2013). EDGE detection techniques using Hough transform. International Journal of Emerging Technology and Advanced Engineering, 3(6), 333-337.
[11]. Savant, S. (2014). A review on edge detection techniques for image segmentation. International Journal of Computer Science and Information Technologies, 5(4), 5898-5900.

[12]. Jain, P., \& Tyagi, V. (2016). A survey of edgepreserving image denoising methods. Information Systems Frontiers, 18(1), 159-170. https://en.wikipedia.org/wiki/Kirsch_operator. https://doi.org/10.1007/s10796-014-9527-0

[13]. Gupta, S., Gupta, C., \& Chakarvarti, S. K. (2013). Image Edge Detection: A Review. International Journal of Advanced Research in Computer Engineering \& Technology (IJARCET), 2(7).

[14]. Rashidha, R., \& Simon, P. (2016). An adaptivesize median filter for impulse noise removal using neural network-based detector. International Journal of Signal and Imaging Systems Engineering, 9(4-5), 305-310. https://doi.org/10.1504/IJSISE.2016.078254

\section{Cite this article as :}

Rana Riad K. Al-Taie, Basma Jumaa Saleh, Lamees Abdalhasan Salman, "Image Edge-Segmentation Techniques : A Review", International Journal of Scientific Research in Science, Engineering and Technology (IJSRSET), Online ISSN : 2394-4099, Print ISSN : 2395-1990, Volume 8 Issue 5, pp. 252-257, September-October 2021. Available at doi : https://doi.org/10.32628/IJSRSET218528 Journal URL : https://ijsrset.com/IJSRSET218528 